TITLE:

Unitary Representations of the Hyperfinite Heisenberg Group and the Logical Extension Methods in Physics

\author{
$\operatorname{AUTHOR}(\mathrm{S}):$ \\ OJIMA, IZUMI; OZAWA, MASANAO
}

CITATION:

OJIMA, IZUMI ... [et al]. Unitary Representations of the Hyperfinite Heisenberg Group and the Logical Extension Methods in Physics. 数理解析研究所講究録 1994, 885: 60-91

ISSUE DATE:

1994-09

URL:

http://hdl.handle.net/2433/84294

RIGHT: 


\title{
Unitary Representations of the Hyperfinite Heisenberg Group and the Logical Extension Methods in Physics
}

\author{
IzUmi OJIMA and Masanao Ozawa* \\ Research Institute for Mathematical Sciences \\ Kyoto University, Kyoto 606, Japan \\ and \\ ${ }^{*}$ Department of Mathematics, College of General Education \\ Nagoya University, Nagoya 464, Japan
}

\begin{abstract}
In order to provide a general framework for applications of nonstandard analysis to quantum physics, the hyperfinite Heisenberg group, which is a finite Heisenberg group in nonstandard universe, is formulated and its unitary representations are examined. The ordinary Schrödinger representation of the Heisenberg group is obtained by a suitable standardization of its internal representation. As an application, a nonstandard-analytical proof of noncommutative Parseval's identity based on the orthogonality relations for unitary representations of finite groups is shown. This attempt is placed in a general framework, called the logical extension methods in physics, which aims at the systematic applications of methods of foundations of mathematics to extending physical theories. The program and the achievement of the logical extension methods are explained in some detail.
\end{abstract}




\section{Introduction}

In recent years the interplay between foundations of quantum mechanics and physics of macroscopic or mesoscopic systems has made evident the demand for thorough understanding of the mutual relationship between quantum mechanics and classical mechanics. The developments in such experimental researches as microfabrication techniques, quantum optics, precision measurements, as well as their applications to the optical communication and the gravitational-wave detection have opened a new domain of scale in applications of quantum mechanics different from the traditional scale of atomic physics [1]. These experimental attempts have prompted the research in the quantum theory of non-ideal measurements of continuous observables incorporating with introduction of the deep ideas from probability theory, mathematical statistic, and information theory [2].

Now, one of the major problems in mathematical physics required from these recent developments is to provide a suitable mathematical framework for applying quantum mechanics to more macroscopic objects than the traditional scale of atomic physics in order to make it possible to describe interactions between macroscopic objects and microscopic objects. A difficulty in this kind of descriptions has been well known, exemplied by the famous paradox of Schrödinger's cat, which caricatures the absurdity arising when quantum mechanics is applied to a phenomenon including a very large scale difference. The traditional prescription of the correspondence principle between quantum mechanics and classical mechanics by no means works for this problem, since taking of the limit as $\hbar \rightarrow 0$ changes the whole system, and no microscopic structures are maintained after taking limit. Moreover, the $\mathrm{C}^{*}$-algebraic approach, which has been considered to give one of the most general mathematical framework for quantum field theory and statistical mechanics [4], does not seem to 
work so well in this context: According to Ozawa [5], it is not possible to describe a measuring interaction between a microscopic system and a macroscopic apparatus by the standard notions of $\mathrm{C}^{*}$-dynamical system (if one sticks to the conventional idea of describing this interaction process in use of a 1-parameter automorphism group.) To attain a consistent description of number-phase uncertainty relations (which is an important issue in the theoretical description of quantum optical processes), Ozawa [6] attempted to incorporate, into quantum mechanics, the number system containing the infinities or infinitesimals. It is remarkable in this approach that quantum mechanics can be conveniently formulated in the nonstandard universe just on the hyperfinite lattice in a discretized phase space with infinitesimal intervals, whose unit cells have just the volume equal to the Planck constant $h=2 \pi \hbar$. Although this looks simply like the repetition of the naive intuitive picture of Planck's quantization method $[7,8]$, it will turn out to provide us with a more general and flexible framework of quantum theory, when it is combined with a sophisticated mathematical methods of nonstandard analysis. Then, the main purpose of the present paper is to provide a general framework for the attempts of extending quantum mechanics to the extended number system, and to explain a general feature of our method, the logical extension method in physics, which aims at the systematic applications of methods of foundations of mathematics to extending physical theories.

To this end, it may be instructive to give first an overview of this mathematical method of logical extension. Originally, methods of foundations of mathematics have been developed mainly for producing metatheorems which are theorems on axioms, inferences, proofs, models, and so on, but not for producing theorems on ordinary objects of mathematics. This is a main reason why there has been very little interest in the interplay between physics and foundations of mathematics. However, in the last three decades, foundations of mathematics have developed two methods useful 
for ordinary mathematics: nonstandard analysis and Boolean-valued analysis.

In 1960 Robinson found that the methods of nonstandard models in mathematical logic provides a suitable framework of calculus with infinitesimal and infinite numbers [9]. His method, nonstandard analysis, has been developed nowadays in various fields of mathematics [10], in particular, in probability theory and stochastic analysis [11]. By the way, in 1966, three years after Cohen proved the independence of continuum hypothesis by the method of forcing [12], Scott and Solovay invented Boolean-valued models of set theory [13] which reformulates Cohen's forcing in a simple framework. Although the original aim of the Boolean-valued model is independence proofs, its usefulness for ordinary mathematics has been pointed out by Scott [14]. This idea was realized in 1978 when Takeuti [15] instituted the Boolean-valued analysis, the systematic applications of Boolean-valued models to mathematical problems. In subsequent years, the Boolean-valued analysis has been developed in algebra, harmonic analysis, and operator algebras [16]-[18].

In the context of mathematics, the motivation for the logical extension methods came in 1985 from the following two results in Boolean-valued analysis and nonstandard analysis: the "algebras of all bounded operators on Hilbert spaces" in a Boolean-valued universe is functorially equivalent to the "type I von Neumann algebras of all decomposable operators on direct integral Hilbert spaces" in the standard universe $[17,18]$, and that a "matrix algebra" in the nonstandard universe naturally gives rise to a "type $\mathrm{II}_{1}$ factor" in the standard universe [19]. The former result has led to the following logical analysis of an extension of quantum mechanics used in a measurement theory $[20,21]$ : "quantum mechanics with a continuous superselection rule" in the standard universe is equivalent to "quantum mechanics" in the Boolean-valued uiverse plus "averaging over the Boolean truth-values" [22]. The latter result in nonstandard analysis suggests a possibility to develop quatum mechanics only with finite 
matrices in the nonstandard universe, and has led to the number-phase quantization [23] which realizes the ideal limit of the Pegg-Barnet phase operator [24].

The general idea of the logical extension methods as a general paradigm for extending physical theories arose from the discussions between present authors since 1987. Through their collaboration in 1991, the idea of quantum mechanics on the hyperfinite lattice in the nonstandard universe has been crystalized into the general framework unifying the quantum and classical mechanics in various situations, on the basis of the hyperfinite Heisenberg group and its unitary representations (hyperfinite harmonic analysis). In this context, the noncommutative Parseval's identity for the canonical commutation relation can be proved in a very natural way by transferring the orthogonality relations for the unitary representations of compact or finite groups [25]. The perspectives of the program and the achievement in the logical extension method in physics is summarized in Section 3.

In Section 2, a general framework for applications of nonstandard analysis to quantum physics is developed. While the discovery of Loeb measures [26] has yielded the considerable developments of nonstandard analysis in probability theory and stochastic analysis, the applications to quantum physics have been just sporadic [27]. One reason for this appears to be the absence of the universal target of research which can be described simply in the language for the nonstandard universe and yet contains sufficiently complicated structures of the research objects. It goes without saying that the hyperfinite probability space is the target in probability theory and fulfills ideally these conditions. In this paper, we propose the hyperfinite Heisenberg group as a universal target in the applications of nonstandard analysis to quantum physics. In Subsections 2.1 and 2.2, necessary preliminaries for nonstandard analysis are given. In Subsection 2.3, the hyperfinite Heisenberg group is defined together with its internal Schrödinger representation. In Section 2.4, it is shown that an appropriate 
standardization of the internal Schrödinger representation naturally gives rise to the standard Schrödinger representation of the standard Heisenberg group. As an application of the present framework, a rigorous nonstandard-analytical proof is carried out, in Section 2.5, of noncommutative Parseval's identity for the representation of the canonical commutation relation (CCR).

\section{Unitary representations of the hyperfinite Heisenberg group}

\subsection{Nonstandard universe}

For any set $X, P(X)$ stands for the set of all subsets of $X$, called the power set of $X$. The superstructure over $X$, denoted by $V(X)$, is defined by the following recursion:

$$
\begin{gathered}
V_{0}(X)=X, \quad V_{n+1}(X)=V_{n}(X) \cup P\left(V_{n}(X)\right) \\
V(X)=\bigcup_{n \in \omega} V_{n}(X)
\end{gathered}
$$

where $\omega$ is the set of finite ordinals. The set $X$ is called a base set if $\emptyset \notin X$ and for all $x \in X, x \cap V(X)=\emptyset$. It is shown that any set $X$ can be easily replaced by a base set of the same size [28, p. 287], and we always assume that $X$ is a base set. An element of $X$ is called an atom relative to $V(X)$, and an element of $V(X) \backslash X$ a set relative to $V(X)$. The language which describes $V(X)$ is the language $\mathcal{L}(V(X))$ of set theory augmented by the set $\left\{C_{u} \mid u \in V(X)\right\}$ of constant symbols; $C_{u}$ denotes $u$, and we will write $\phi\left[u_{1}, \ldots, u_{n}\right]$ for $\phi\left(C_{u_{1}}, \ldots, C_{u_{n}}\right)$. Thus any formula in $\mathcal{L}(V(X))$ is constructed from the symbols for logical connectives $\neg, \wedge, \vee, \Rightarrow, \Leftrightarrow$, quantifiers $\forall, \exists$, individual variables $x^{\prime}, x^{\prime \prime}, \ldots$, individual constants $C_{u}(u \in V(X)$, and two binary predicate constants $=, \epsilon$. An individual variable $x$ in a formula $\phi$ is called bound when $\phi$ has a quantifier $(\forall x)$ or $(\exists x)$ which is effective on $x$, and called free otherwise. A formula in which every variable is bound is called a statement. We 
will use the following abbreviations, called bounded quantifiers: $(\forall x \in y) \phi$ means $(\forall x)[x \in y \Rightarrow \phi],(\exists x \in y) \phi$ means $(\exists x)[x \in y \wedge \phi]$. A $\Sigma_{0}$-formula is a formula constructed from atomic formulas using connectives and bounded quantifiers.

The interpretation of the statements in $\mathcal{L}(V(X))$ relative to $V(X)$ is given by the following rules:

(1) $C_{u}=C_{v}$ is true if and only if $u$ and $v$ are identical.

(2) $C_{u} \in C_{v}$ is true if and only if $u$ is an element of $v$.

(3) $\neg \phi$ is true if and only if $\phi$ is not true.

(4) $\left(\phi_{1}\right) \wedge\left(\phi_{2}\right)$ is true if and only if both $\phi_{1}$ and $\phi_{2}$ are true.

(5) $\left(\phi_{1}\right) \vee\left(\phi_{2}\right)$ is true if and only if either $\phi_{1}$ or $\phi_{2}$ is true.

(6) $\left(\phi_{1}\right) \Rightarrow\left(\phi_{2}\right)$ is true if and only if $\phi_{1}$ is not true or $\phi_{2}$ is true.

(7) $(\forall x) \phi(x)$ is true if and only if $\phi\left[C_{u}\right]$ is true, for all $u$ in $V(X)$.

(8) $(\exists x) \phi(x)$ is true if and only if there is some $u$ in $V(X)$ such that $\phi\left[C_{u}\right]$ is true.

We will write $V(X) \vDash \phi$ when $\phi$ is true under this interpretation.

A nonstandard universe is a triple $\langle V(X), V(Y), \star\rangle$ consisting of superstructures $V(X), V(Y)$ and a map $\star: V(X) \rightarrow V(Y)$ satisfying the following three conditions:

(1) $X$ and $Y$ are infinite base sets.

(2) (Transfer Principle) The map $\star: a \mapsto{ }^{\star} a$ is an injective mapping from $V(X)$ into $V(Y)$, and for any $\Sigma_{0}$-formula $\phi\left(x_{1}, \ldots, x_{n}\right)$ in $\mathcal{L}$,

$$
V(X) \vDash \phi\left[u_{1}, \ldots, u_{n}\right] \text { if and only if } V^{r}(Y){ }^{\prime}=\phi\left[{ }^{\star} u_{1}, \ldots,{ }^{\star} u_{n}\right] \text {, }
$$

for any $u_{1}, \ldots, u_{n}$ in $V(X)$.

(3) ${ }^{\star} X=Y$. 
In this case, $u \in V(Y)$ is called standard if there is $x \in V(X)$ such that $u={ }^{\star} x$, and called internal if there is $x \in V(X)$ such that $u \in{ }^{\star} x$. A nonstandard universe $\langle V(X), V(Y), \star\rangle$ is called countably saturated, or $\aleph_{1}$-saturated, if it satisfies the following condition:

(4) (Saturation Principle) Any countable sequence of internal sets $A_{n} \in V(Y) \backslash Y$ with the finite intersection property has a nonempty intersection.

A mapping from $V(X)$ to $V(Y)$ satisfying the Transfer Principle described in (2) is called generally a bounded elementary embedding, and $V(Y)$ a bounded elementary extension.

The following theorem is a model-theoretic foundation of nonstandard analysis.

Theorem 2.1. (Existence of Nonstandard Universes) There exists a countably saturated nonstandard universe $\langle V(X), V(Y), \star\rangle$.

For the proof, we refer to [28, p. 268, Theorem 4.4.5].

Let $A$ be a set relative to $V(X)$. From the Saturation Principle, we can show that, if $A \in V(X)$ is an infinite set, ${ }^{\sigma} A$ defined by

$$
{ }^{\sigma} A=\left\{{ }^{\star} a \mid a \in A\right\}
$$

is a proper subset of ${ }^{\star} A$. Thus ${ }^{\star} A$ for any infinite set $A$ relative to $V(X)$ is an extension of $A$, and called the nonstandard extension of $A$. By renaming the elements of $\mathrm{Y}$, we can assume without loss of generality that $X$ is a subset of of ${ }^{\star} X$ and ${ }^{\star} x=x$ for each $x \in X$.

Given any subset $U \subset V(X)$, define ${ }^{\star} U$ by

$$
{ }^{\star} U=\bigcup_{n \in \omega}{ }^{\star}\left(U \cap V_{n}(X)\right)
$$


Let $F(X)$ be the set of finite sets relative to $V(X)$. An element of ${ }^{\star} F(X)$ is called a hyperfinite set. Hyperfinite sets have the same formal properties as the finite sets relative to $V(X)$. The set ${ }^{\star} V(X)$ is the set of all internal sets.

\subsection{Infinitesimal analysis}

In what follows, we will develop fragments of infinitesimal analysis based on a countably saturated nonstandard universe $\left\langle V(\mathrm{~N}), V\left({ }^{\star} \mathrm{N}\right), \star\right\rangle$. The set $\mathrm{N}$ of natural numbers is usually defined as the set $\omega$ of finite ordinal numbers, but since $\mathrm{N}$ is not a base set, we redefine $\mathrm{N}$ as the base set of all $\{\omega \backslash n\}$ with $n \in \omega$, so that the $n$-th natural number $(n \in \omega)$ is $\{\omega \backslash n\}$. Note that all mathematical objects having been necessary for physics are in $V(\mathrm{~N})$; for example, natural numbers are in $V_{0}(\mathrm{~N})$, integers are in $V_{2}(\mathrm{~N})$, rational numbers are in $V_{5}(\mathrm{~N})$, real numbers are in $V_{6}(\mathrm{~N})$, and complex numbers are in $V_{8}(\mathrm{~N})$. We have also $\mathrm{N} \in V_{1}(\mathrm{~N})$, the set of integers $\mathrm{Z} \in V_{3}(\mathrm{~N})$, the set of rationals $Q \in V_{6}(\mathrm{~N})$, the set of reals $\mathrm{R} \in V_{7}(\mathrm{~N})$, the set of complex numbers $\mathrm{C} \in V_{9}(\mathrm{~N})$, Euclidean space $\mathrm{R}^{n} \in V_{4+2 n}(\mathrm{~N})$, Schwartz space $\mathcal{S}(\mathrm{R}) \in V_{11}(\mathrm{~N})$, space of tempered distributions $\mathcal{S}^{\prime}(\mathrm{R}) \in V_{14}(\mathrm{~N})$, Hilbert space $L^{2}(\mathrm{R}) \in V_{12}(\mathrm{~N})$, and for $n>1, \mathcal{S}\left(\mathbf{R}^{n}\right) \in V_{7+2 n}(\mathrm{~N}), \mathcal{S}^{\prime}\left(\mathbf{R}^{n}\right) \in V_{10+2 n}(\mathrm{~N}), L^{2}\left(\mathrm{R}^{n}\right) \in V_{8+2 n}(\mathrm{~N})$, and the set of all von Neumann algebras acting on $L^{2}\left(\mathrm{R}^{n}\right)$ is in $V_{13+2 n}(\mathrm{~N})$.

The elements of ${ }^{\star} \mathbf{R}$ are called hyperreal numbers. We will assume $\mathbf{R} \subset{ }^{\star} \mathbf{R}$ by identifying $x \in \mathrm{R}$ with ${ }^{\star} x \in{ }^{\star} \mathrm{R}$. By the Transfer Principle, ${ }^{\star} \mathrm{R}$ is a proper ordered field extension of ${ }^{\star} \mathbf{R}$. A hyperreal number $x$ is called infinite if $|x|>n$ for any $n \in \mathbf{N}$, finite, write $|x|<\infty$, if there is some $n \in N$ such that $|x|<n$, and infinitesimal if $|x|<1 / n$ for any $n \in \mathrm{N}$. The elements of ${ }^{\star} \mathrm{C}$ is called hypercomplex numbers; we assume $\mathrm{C} \subset{ }^{\star} \mathrm{C}$ by identifying $x \in \mathrm{C}$ with ${ }^{\star} x \in{ }^{\star} \mathrm{C}$. For any $x, y \in{ }^{\star} \mathrm{C}$, we will write $x \approx y$ if $|x-y|$ is infinitesimal, $x=O(y)$ if $y \neq 0$ and $|x / y|$ is finite, and $x \asymp y$ if $x, y \neq 0$ and both $|x / y|$ and $|y / x|$ are finite. For any finite hyperreal number $x$, 
there is a unique real number $r$ such that ${ }^{\star} r \approx x$; this $r$ is called the standard part of $x$ and denoted by ${ }^{\circ} x$.

Any function $f$ from $X$ to $Y$ is extended to an internal function ${ }^{\star} f$ from ${ }^{\star} X$ to ${ }^{\star} Y$. A sequence $a_{n} \in \mathbf{C}(n \in \mathrm{N})$ is extended to an internal sequence ${ }^{\star} a_{\nu} \in{ }^{\star} \mathbf{C}$ $\left(\nu \in{ }^{\star} \mathrm{N}\right)$, so that $\lim _{n \rightarrow \infty} a_{n}=a$ if and only if ${ }^{\star} a_{\nu} \approx a$ for all $\nu \in{ }^{\star} \mathrm{N} \backslash \mathrm{N}$.

Let $\mathcal{X}$ be an internal normed linear space with norm $\|\cdot\|$. The principal galaxy fin $(\mathcal{X})$ and the principal monad $\mu(\mathcal{X})$ are defined by

$$
\begin{aligned}
\operatorname{fin}(\mathcal{X}) & =\left\{x \in \mathcal{X} \mid\|x\|_{2}<\infty\right\} \\
\mu(\mathcal{X}) & =\left\{x \in \mathcal{X} \mid\|x\|_{2} \approx 0\right\}
\end{aligned}
$$

Then both of them are linear spaces over $\mathrm{C}$. The nonstandard hull of $\mathcal{X}$ is the quotient linear space $\hat{\mathcal{X}}=\operatorname{fin}(\mathcal{X}) / \mu(0)$ equipped with the norm given by

$$
\left\|{ }^{\circ} x\right\|={ }^{\circ}\|x\|
$$

for all $x \in \operatorname{fin}(\mathcal{X})$, where ${ }^{\circ} x=x+\mu(0)$. Then it is shown by the Saturation Principle that $\hat{\mathcal{X}}$ is a Banach space, which is called the nonstandard hull of $\mathcal{X}$. For an normed linear space $\mathcal{Y}$, the nonstandard hull of $\mathcal{X}={ }^{\star} \mathcal{Y}$ is usually denoted by $\hat{\mathcal{Y}}$.

Let $\mathcal{H}$ be an internal Hilbert space with inner product $\langle\cdot \mid \cdot\rangle$. Then the nonstandard hull $\hat{\mathcal{H}}$ is also a Hilbert space with inner product given by

$$
\left\langle\left.{ }^{\circ} x\right|^{\circ} y\right\rangle={ }^{\circ}\langle x \mid y\rangle
$$

for any $x, y \in \operatorname{fin}(\mathcal{H})$. Let ${ }^{\star} \mathcal{L}(\mathcal{H})$ be the internal algebra of bounded operators on $\mathcal{H}$. Then ${ }^{\star} \mathcal{L}(\mathcal{H})$ is an internal $C^{*}$-algebra, and it is easy to see that its nonstandard hull $\star \widehat{\mathcal{L}(\mathcal{H})}$ is also a $\mathrm{C}^{*}$-algebra. Let $T: \mathcal{H} \rightarrow \mathcal{H}$ be an internal bounded operator such that the bound $\|T\|$ is finite, i.e., $T \in \operatorname{fin}\left({ }^{\star} \mathcal{L}(\mathcal{H})\right)$. Then, $T$ leaves $f \mathrm{in}(\mathcal{H})$ and $\mu(0)$ invariant, and induces a linear map $\hat{T}$ on $\hat{\mathcal{H}}$ by the relation $\hat{T}(x+\mu(0))=T x+\mu(0)$. 
It is easy to see that $\|\hat{T}\|={ }^{\circ}\|T\|$, and that the correspondence ${ }^{\circ} T \rightarrow \hat{T}$ is a faithful *-representation of $\star \widehat{\mathcal{L}(\mathcal{H})}$ on $\hat{\mathcal{H}}$. The operator $\hat{T}$ will be called the nonstandard hull of $T$.

\subsection{Hyperfinite Heisenberg group}

Let $K$ be a fixed nonstandard natural number, i.e., $K \in{ }^{\star} \mathrm{N} \backslash \mathrm{N}$. Let $\mathrm{K}$ be the ring of residue classes of internal integers modulo $K$, i.e., $\mathbf{K}={ }^{\star} \mathrm{Z} / K^{\star} \mathbf{Z}$. For $k, k^{\prime} \in \mathbf{K}$, $k \oplus k^{\prime}$ stands for the sum of $k$ and $k^{\prime}$ in $\mathrm{K}, k \otimes k^{\prime}$ the product of $k$ and $k^{\prime}$, and $\Theta k$ the minus $k$. We always assume that $\mathrm{K}$ is represented by a complete system of incongruent residues to modulus $K$. The hypercomplex number $e^{2 \pi i k / K}$ is unambiguously determined independent of the choice of the complete system, and we have $e^{2 \pi i\left(k \oplus k^{\prime}\right) / K}=e^{2 \pi i\left(k+k^{\prime}\right) / K}$ and $e^{2 \pi i\left(k \otimes k^{\prime}\right) / K}=e^{2 \pi i k k^{\prime} / K}$ for all $k, k^{\prime} \in \mathbf{K}$.

Let $R(\mathrm{~K})$ be the internal $K$-dimensional linear space of ${ }^{\star} \mathrm{C}$-valued internal functions on $\mathrm{K}$. Let $\Delta x$ be a fixed positive hyperreal number. Denote by $R(\mathrm{~K}, \Delta x)$ the internal unitary space consisting of the internal linear space $R(\mathrm{~K})$ and the internal inner product $\langle\cdot \mid \cdot\rangle$ defined by

$$
\langle f \mid g\rangle=\sum_{k \in \mathrm{K}} \overline{f(k)} g(k) \Delta x
$$

for all $f, g \in R(\mathbf{K}, \Delta x)$, where $\sum$ denotes the internal sum. The norm of $f \in$ $R(\mathrm{~K}, \Delta x)$ is defined by $\|f\|=\langle f \mid f\rangle^{1 / 2}$. Let $\delta_{k}$ be the internal function such that

$$
\delta_{k}\left(k^{\prime}\right)=\frac{1}{\Delta x} \delta_{k, k^{\prime}}
$$

for all $k^{\prime} \in \mathrm{K}$, where $\delta_{k, k^{\prime}}$ stands for Kronecker's delta. Then $\left\{\delta_{k} \mid k \in \mathrm{K}\right\}$ is a basis of $R(\mathrm{~K}, \Delta x)$ such that

$$
\left\langle\delta_{k} \mid f\right\rangle=f(k)
$$


for all $f \in R(\mathbf{K}, \Delta x)$ and $k \in \mathbf{K}$. For any natural number $n$, the space $R\left(\mathrm{~K}^{n},(\Delta x)^{n}\right)$ is defined analogously, so that $R\left(\mathrm{~K}^{n},(\Delta x)^{n}\right)$ is internally isometrically isomorphic to $\bigotimes_{j=1}^{n} R(\mathrm{~K}, \Delta x)$.

The hyperfinite Heisenberg group based on $K$ is the group $\mathrm{H}$ with the underlying set $\mathrm{K} \times \mathbf{K} \times \mathbf{K}$ whose group operation is

$$
(k, l, m)\left(k^{\prime}, l^{\prime}, m^{\prime}\right)=\left(k \oplus k^{\prime}, l \oplus l^{\prime}, m \oplus m^{\prime} \oplus k \otimes l^{\prime}\right)
$$

Then the unit is $(0,0,0)$, and the inverse of $(k, l, m)$ is $(\ominus k, \ominus l, \ominus m \oplus k \otimes l)$.

For any $(k, l, m) \in \mathrm{H}$, define the operator $W(k, l, m)$ on $R(\mathrm{~K})$ by

$$
W(k, l, m) f\left(k^{\prime}\right)=e^{2 \pi i\left(m+l k^{\prime}\right) / K} f\left(k^{\prime} \oplus k\right)
$$

for all $f \in R(\mathbf{K})$, and $k^{\prime} \in \mathbf{K}$.

Proposition 2.2. The map $W:(k, l, m) \mapsto W(k, l, z)$ is an internal irreducible unitary representation of the Heisenberg group $\mathrm{H}$ on $R(\mathrm{~K}, \Delta x)$.

Proof. Follows from Transfer Principle.

We call the representation $W$ the internal Schrödinger representation of H. Denote by $\hat{R}(\mathrm{~K}, \Delta x)$ the nonstandard hull of $R(\mathrm{~K}, \Delta x)$, and by $\hat{W}(k, l, m)$ the nonstandard hull of $W(k, l, m)$ on $\hat{R}(\mathrm{~K}, \Delta x)$. The definition of $\hat{W}(k, l, m)$ depends on $\Delta x$, and we will denote it by $\hat{W}_{\Delta x}(k, l, m)$ when the dependence of $\Delta x$ is to be explicit.

Proposition 2.3. The map $\hat{W}:(k, l, m) \mapsto \hat{W}(k, l, m)$ is an unitary representation of $\mathrm{H}$ on $\hat{R}(\mathrm{~K}, \Delta x)$.

Proof. An immediate consequence from the fact that the correspondence $W(k, l, m) \rightarrow \hat{W}(k, l, m)$ is an algebraic homomorphism. 
For any $\Delta x$ and $\Delta x^{\prime}$, the scalar multiplication on $R(\mathrm{~K})$ by $\sqrt{\Delta x / \Delta x^{\prime}}$ is an internal unitary transformation from $R(\mathrm{~K}, \Delta x)$ onto $R\left(\mathrm{~K}, \Delta x^{\prime}\right)$, which implements the internal unitary equivalence between $W$ on $R(\mathbf{K}, \Delta x)$ and $W$ on $R\left(\mathbf{K}, \Delta x^{\prime}\right)$.

Proposition 2.4. For any positive hyperreals $\Delta x$ and $\Delta x^{\prime}$, if $\Delta x \asymp \Delta x^{\prime}$ then $\hat{W}_{\Delta x}$ on $\hat{R}(\mathbf{K}, \Delta x)$ and $\hat{W}_{\Delta x^{\prime}}$ on $\hat{R}\left(\mathbf{K}, \Delta x^{\prime}\right)$ are unitarily equivalent.

Proof. Let $U$ be the internal unitary transformation from $R(\mathrm{~K}, \Delta x)$ onto $R\left(\mathrm{~K}, \Delta x^{\prime}\right)$ defined by $U f=\sqrt{\Delta x / \Delta x^{\prime}} f$. If $\Delta x \asymp \Delta x^{\prime}$, then $\sqrt{\Delta x / \Delta x^{\prime}}$ is finite, and the nonstandard hull $\hat{U}$ of $U$ is a unitary transformation from $\hat{R}(\mathbf{K}, \Delta x)$ onto $\hat{R}\left(\mathbf{K}, \Delta x^{\prime}\right)$, which implements the unitary equivalence between $\hat{W}_{\Delta x}$ and $\hat{W}_{\Delta x^{\prime}}$.

\subsection{The Schrödinger representation}

Let $S$ be a nonstandard natural number. In this section, we assume $K=4 S^{2}$, $\Delta x=(2 S)^{-1}=K^{-1 / 2}, \epsilon=\Delta x / 2$ and identify $\mathrm{K}$ with complete sỹstem

$$
\left\{-2 S^{2}+1, \ldots,-1,0,1, \ldots, 2 S^{2}\right\}
$$

of incongruent residues to modulus $K$.

Consider $\mathbf{R}^{3}$ with coordinates $(p, q, t)$. We make $\mathbf{R}^{3}$ into a locally compact group with group law

$$
(p, q, t)\left(p^{\prime}, q^{\prime}, t^{\prime}\right)=\left(p+p^{\prime}, q+q^{\prime}, t+t^{\prime}+p q^{\prime}\right)
$$

We call this group the Heisenberg group and denote it by $\mathbf{H}_{1}$; in [29] this is called the polarized Heisenberg group.

Let $n$ be a natural number. Denote by $L^{p}\left(\mathrm{R}^{n}\right)$ the $L^{p}$ space with respect to Lebesgue measure, $\|f\|_{p}$ is the $L^{p}$ norm of $f$. The inner product is defined by

$$
\langle f \mid g\rangle=\int_{\mathbf{R}^{n}} \overline{f(x)} g(x) d x
$$


for all $f, g \in L^{2}\left(\mathbf{R}^{n}\right)$.

Let $h$ be a positive real number. The Schrödinger representation of $\mathbf{H}_{1}$ with Planck's constant $h$ is the strongly continuous unitary representation $\rho_{h}$ of $\mathrm{H}_{1}$ on $L^{2}(\mathbf{R})$ defined by

$$
\rho_{h}(p, q, t) f(x)=e^{2 \pi i(t+q x) / h} f(x+p)
$$

for all $f \in L^{2}(\mathbf{R})$.

Denote by $L^{p}\left({ }^{\star} \mathbf{R}^{n}\right)$ the nonstandard extension of $L^{p}\left(\mathbf{R}^{n}\right)$ with internal norm $\|\cdot\|_{p}$. The internal inner product is denoted by $\langle\cdot \mid \cdot\rangle$, which satisfies

$$
\langle f \mid g\rangle=\int_{\star_{\mathbf{R}^{n}}} \overline{f(x)} g(x) d x
$$

for all $f, g \in L^{2}\left({ }^{\star} R^{n}\right)$, where the integral is internal Lebesgue integral. We will denote the nonstandard hull of $L^{p}\left({ }^{\star} \mathbf{R}^{n}\right)$ by $\hat{L}^{p}\left({ }^{\star} \mathbf{R}^{n}\right)$. For $f \in L^{p}\left(\mathbf{R}^{n}\right)$, we will identify ${ }^{\circ \star} f$ with $f$ for simplicity of notations, and accordingly identify the subset $\left\{{ }^{\circ} f \mid f \in{ }^{\sigma}\left(L^{p}\left(\mathbf{R}^{n}\right)\right)\right\}$ of $\hat{L}^{p}\left(\mathbf{R}^{n}\right)$ with $L^{p}\left(\mathbf{R}^{n}\right)$.

For any $k \in \mathbf{K}$, consider the internal intervals $I_{k}=(k \Delta x-\epsilon, k \Delta x+\epsilon]$. Let $\mathcal{F}$ be the internal $\sigma$-subfield of the internal Borel $\sigma$-field $\mathcal{B}\left({ }^{\star} \mathrm{R}\right)$ generated by all $I_{k}(k \in \mathrm{K})$, and $L^{2}(\mathcal{F})$ the internal closed subspace of $L^{2}\left({ }^{\star} R\right)$ consisting of internal $\mathcal{F}$-measurable functions. For any $k \in \mathrm{K}$, let $\Delta_{k}$ be the.indicator function of the internal interval $I_{k}$, i.e., $\Delta_{k}(x)=1$ if $x \in I_{k}$, and $\Delta_{k}(x)=0$ otherwise. Then $L^{2}(\mathcal{F})$ is internally spanned by $\left\{\Delta_{k} \mid k \in \mathrm{K}\right\}$, and we have

$$
\left\langle\Delta_{k} \mid \Delta_{k^{\prime}}\right\rangle=\delta_{k, k^{\prime}} \Delta x
$$

We will denote the internal closed subspace $\bigotimes_{j=1}^{n} L^{2}(\mathcal{F})$ of $L^{2}\left({ }^{\star} \mathrm{R}^{n}\right)$ by $L^{2}\left(\mathcal{F}^{n}\right)$.

Let $\mathcal{U}$ be a linear map from $R\left(\mathrm{~K}^{n}\right)$ to $L^{2}\left(\mathcal{F}^{n}\right)$ such that

$$
\mathcal{U}=\sum_{k_{1}, \ldots, k_{n} \in \mathrm{K}} f\left(k_{1}, \ldots, k_{n}\right) \Delta_{k_{1}} \cdots \Delta_{k_{n}}
$$


for all $f \in R\left(\mathrm{~K}^{n}\right)$. Then it is easy to see that $\mathcal{U}$ is an internal unitary transformation from $R\left(\mathrm{~K}^{n},(\Delta x)^{n}\right)$ onto $L^{2}\left(\mathcal{F}^{n}\right)$, and that $\mathcal{U}\left(\delta_{k_{1}} \cdots \delta_{k_{n}}\right)=(\Delta x)^{-n} \Delta_{k_{1}} \cdots \Delta_{k_{n}}$. For any $(k, l, m) \in \mathbf{H}$, define the internal unitary operator $\pi(W(k, l, m))$ on $L^{2}\left(\mathcal{F}^{n}\right)$ by

$$
\pi(W(k, l, m))=\mathcal{U} W(k, l, m) \mathcal{U}^{-1}
$$

and denote by $\hat{\pi}(W(k, l, m))$ the nonstandard hull of $\pi(W(k, l, m))$. Obviously, the $\operatorname{map}(k, l, m) \rightarrow \pi(W(k, l, m))$ is an internal unitary representation of $\mathbf{H}$ internally unitarily equivalent to $W$, and $(k, l, m) \rightarrow \hat{\pi}(W(k, l, m))$ is a unitary representation of $\mathbf{H}$ unitarily equivalent to $\hat{W}$.

Let $C_{b}\left(\mathrm{R}^{n}\right)$ be the space of bounded complex-valued continuous functions on $\mathrm{R}^{n}$, and $C_{b}\left({ }^{\star} \mathrm{R}^{n}\right)$ the nonstandard extension of $C_{b}\left(\mathbf{R}^{n}\right)$. For any $f \in C_{b}\left({ }^{\star} \mathrm{R}^{n}\right)$, define $\mathcal{S} f \in R\left(\mathrm{~K}^{n}\right)$ by

$$
(\mathcal{S} f)\left(k_{1}, \ldots, k_{n}\right)=f\left(k_{1} \Delta x, \ldots, k_{n} \Delta x\right)
$$

for all $k_{i} \in \mathrm{K}(i=1, \ldots, n)$. Then the map $\mathcal{S}: f \mapsto \mathcal{S} f$ is defined as an internal linear transformation from $C_{b}\left({ }^{\star} \mathrm{R}^{n}\right)$ to $R\left(\mathrm{~K}^{n},(\Delta x)^{n}\right)$. For any $f \in L^{2}\left({ }^{\star} \mathrm{R}^{n}\right)$, define $\mathcal{E} f \in R\left(\mathbf{K}^{n}\right)$ by

$$
(\mathcal{E} f)\left(k_{1}, \ldots, k_{n}\right)=\frac{1}{(\Delta x)^{n}}\left\langle\Delta_{k_{1}} \cdots \Delta_{k_{n}} \mid f\right\rangle=\frac{1}{(\Delta x)^{n}} \int_{I_{k_{1}} \times \cdots \times I_{k n}} f(x) d x
$$

The map $\mathcal{E}: f \mapsto \mathcal{E} f$ is defined as an internal linear transformation from $L^{2}\left({ }^{\star} \mathbf{R}^{n}\right)$ to $R\left(\mathrm{~K}^{n}\right)$. Let $\mathrm{E}$ be the internal projection from $L^{2}\left({ }^{\star} \mathrm{R}^{n}\right)$ onto $L^{2}\left(\mathcal{F}^{n}\right)$. Then $\mathrm{E}=\mathcal{U} \mathcal{E}$. We define $\mathrm{S}: L^{2}\left({ }^{\star} \mathrm{R}^{n}\right) \rightarrow L^{2}\left(\mathcal{F}^{n}\right)$ by $\mathrm{S}=\mathcal{U S}$. Denote by $\hat{\mathrm{E}}$ and $\hat{\mathrm{S}}$ the nonstandard hull of $\mathrm{E}$ and $\mathrm{S}$, respectively.

Lemma 2.5. Let $n$ be a natural number. For any $f \in C_{b}\left(\mathbf{R}^{n}\right) \cap L^{p}\left(\mathbf{R}^{n}\right)$, we have

$$
\left\|\mathrm{S}^{\star} f-{ }^{\star} f\right\|_{p} \approx 0
$$


Proof. Let $n=1$. For any $\nu \in{ }^{\star} \mathrm{N}$, let $f_{\nu}$ be such that

$$
f_{\nu}(x)=\left\{\begin{array}{cl}
{ }^{\star} f\left(\frac{k}{2 \nu}\right) & \text { if } k \in{ }^{\star} \mathrm{N},-2 \nu^{2}<m \leq 2 \nu^{2}, \text { and } \frac{2 k-1}{4 \nu}<x \leq \frac{2 k+1}{4 \nu} \\
0 & \text { otherwise, }
\end{array}\right.
$$

for all $x \in \mathbf{R}$. Then it is easy to see that $f_{\nu}$ is standard for all $\nu \in \mathbf{N}$, and that the standard sequence $\left\{\left\|f_{\nu}-{ }^{\star} f\right\|_{p} \mid \nu \in \mathrm{N}\right\}$ converges to 0 , and hence $\left\|f_{s}-{ }^{\star} f\right\|_{p} \approx 0$. Since $\mathbf{S}^{\star} f=f_{S}$, we have $\left\|\mathbf{S} f-{ }^{\star} f\right\|_{p} \approx 0$. The proof for general $n$ is analogous.

Denote by $K(\mathbf{R})$ the space of complex-valued continuous functions on $\mathbf{R}$ with compact support.

Theorem 2.6. For any $f \in L^{2}\left(\mathrm{R}^{n}\right)$, we have $\left\|\mathrm{E}^{\star} f-{ }^{\star} f\right\|_{2} \approx 0$.

Proof. Let $f \in K(\mathbf{R})$. By the transfer principle and a property of projection, we have $\left\|\mathrm{E}^{\star} f-{ }^{\star} f\right\|_{2} \leq\left\|\mathrm{S}^{\star} f-{ }^{\star} f\right\|_{2}$, and hence by Lemma $2.5\left\|\mathrm{E}^{\star} f-{ }^{\star} f\right\|_{2} \approx 0$. It follows that $\hat{\mathbf{E}} f=f$ for all $f \in K(\mathrm{R})$. By the density of $K(\mathrm{R})$ in $L^{2}(\mathbf{R})$ and continuity of projections, this holds for all $f \in L^{2}(\mathbf{R})$. Thus we have $\left\|\mathbf{E}^{\star} f-{ }^{\star} f\right\|_{2} \approx 0$ for all $f \in L^{2}(\mathbf{R})$.

From the above theorem, we can embed $L^{2}\left(\mathbf{R}^{n}\right)$ in $\hat{L}^{2}\left(\mathcal{F}^{n}\right)$ as a closed linear subspace by the correspondence between $f \in L^{2}\left(\mathbf{R}^{n}\right)$ and $\hat{\mathbf{E}} f={ }^{\circ}(\mathbf{E} f) \in \hat{L}^{2}\left(\mathcal{F}^{n}\right)$.

Now, let ${ }^{\star} \mathrm{H}_{1}$ be the nonstandard extension of $\mathrm{H}_{1}$. For any $(p, q, t),\left(p^{\prime}, q^{\prime}, t^{\prime}\right) \in$ ${ }^{\star} \mathrm{H}_{1}$, we write $(p, q, t) \approx\left(p^{\prime}, q^{\prime}, t^{\prime}\right)$, if $p \approx p^{\prime}, q \approx q^{\prime}$, and $t \approx t^{\prime}$, and write $|(p, q, t)|<$ $\infty$, if $|p|<\infty,|q|<\infty$, and $|t|<\infty$. Let $\mathrm{fin}\left({ }^{\star} \mathrm{H}_{1}\right)$ be the principal galaxy of ${ }^{\star} \mathrm{H}_{1}$, and $\mu\left({ }^{\star} \mathrm{H}_{1}\right)$ the principal monad of ${ }^{\star} \mathrm{H}_{1}$, i.e.,

$$
\begin{aligned}
\operatorname{fin}\left({ }^{\star} \mathrm{H}_{1}\right) & =\left\{(p, q, t) \in{ }^{\star} \mathrm{H}_{1}||(p, q, i) \mid<\infty\right\}, \\
\mu\left({ }^{\star} \mathrm{H}_{1}\right) & =\left\{(p, q, t) \in{ }^{\star} \mathrm{H}_{1} \mid(p, q, t) \approx(0,0,0)\right\} .
\end{aligned}
$$

Then we have the following; for the proof see [9, p. 204]. 
Lemma 2.7. The set $\operatorname{fin}\left({ }^{\star} \mathrm{H}_{1}\right)$ is a subgroup of ${ }^{\star} \mathrm{H}_{1}, \mu\left(\mathrm{H}_{1}\right)$ is a normal subgroup of $\mathrm{fin}\left({ }^{\star} \mathrm{H}_{1}\right)$, and the quotient group $\mathrm{fin}\left({ }^{\star} \mathrm{H}_{1}\right) / \mu\left(\mathrm{H}_{1}\right)$ is isomorphic to $\mathrm{H}_{1}$ under the correspondence $x \mu\left(\mathrm{H}_{1}\right)$ and $x \in \mathrm{H}_{1}$.

We will denote by ${ }^{\star} \rho_{h}$ the nonstandard extension of $\rho_{h}$. Then ${ }^{\star} \rho_{h}$ is an internal unitary representation of ${ }^{\star} \mathrm{H}_{1}$ on $L^{2}(\mathrm{R})$. In the following lemmas, we assume $(k, l, m) \in \mathbf{H}$, and $k^{\prime}, k^{\prime \prime} \in \mathbf{K}$.

Lemma 2.8. We have the following relations.

$$
\begin{aligned}
& \left\langle\Delta_{k^{\prime}}|\pi(W(k, l, m))| \Delta_{k^{\prime \prime}}\right\rangle \\
& \quad=\delta_{k^{\prime} \oplus k, k^{\prime \prime}} e^{2 \pi i\left(m+l k^{\prime}\right) / K} \Delta x, \\
& \left\langle\left.\Delta_{k^{\prime}}\right|^{\star} \rho_{h}\left(k \Delta x, h l \Delta x, h m(\Delta x)^{2}\right) \mid \Delta_{k^{\prime \prime}}\right\rangle \\
& \quad=\left\{\begin{array}{cc}
\delta_{k^{\prime}+k, k^{\prime \prime}} e^{2 \pi i\left(m+l k^{\prime}\right) / K} \frac{\sin \pi(l \Delta x) \Delta x}{\pi(l \Delta x) \Delta x} \Delta x, & \text { if } l \neq 0, \\
\delta_{k^{\prime}+k, k^{\prime \prime}} e^{2 \pi i m / K} \Delta x, & \text { if } l=0,
\end{array}\right.
\end{aligned}
$$

Proof. The assertion follows from routine computations and the transfer principle.

Lemma 2.9. If $|k \Delta x|<\infty$ and $\left|k^{\prime} \Delta x\right|<\infty$, then $k^{\prime} \oplus k=k^{\prime}+k$. If $|k \Delta x|<\infty$ and $\left|k^{\prime \prime} \Delta x\right|<\infty$, then $k^{\prime \prime} \ominus k=k^{\prime \prime}-k$. If $|k \Delta x|<\infty$, then $\ominus k=-k$.

Proof. It follows from the assumptions that $\left|k^{\prime} \Delta x+k \Delta x\right|<\infty$. Thus there is a standard $n \in \mathrm{N}$ such that $-n<\left(k^{\prime}+k\right) \Delta x<n$, and hence $-2 S^{2}<-2 n S<k^{\prime}+k<$ $2 n S<2 S^{2}$. Therefore, we have $k^{\prime} \oplus k=k^{\prime}+k$. The proofs for the other assertions are similar.

Lemma 2.10. Suppose $|k \Delta x|<\infty$. If $\left|k^{\prime} \Delta x\right|<\infty$ or $\left|k^{\prime \prime} \Delta x\right|<\infty$, then

$$
\begin{gathered}
\left|\left\langle\Delta_{k^{\prime}}\left|\pi(W(k, l, m))-{ }^{*} \rho_{h}\left(k \Delta x, h l \Delta x, h m(\Delta x)^{2}\right)\right| \Delta_{k^{\prime \prime}}\right\rangle\right| \\
\quad=\left\{\begin{array}{cl}
\delta_{k^{\prime}+k, k^{\prime \prime}}\left(1-\frac{\sin \pi(l \Delta x) \Delta x}{\pi(l \Delta x) \Delta x}\right) \Delta x, & \text { if } l \neq 0, \\
0 & \text { if } l=0 .
\end{array}\right.
\end{gathered}
$$


Proof. When $\left|k^{\prime} \Delta x\right|<\infty$, the assertion follows immediately from Lemmas 2.8 and 2.9. When $\left|k^{\prime \prime} \Delta x\right|<\infty$, the proof is analogous from the relations $\delta_{k^{\prime} \oplus k, k^{\prime \prime}}=\delta_{k^{\prime}, k^{\prime \prime} \ominus k}$, $\delta_{k^{\prime}+k, k^{\prime \prime}}=\delta_{k^{\prime}, k^{\prime \prime}-k}$, and $k^{\prime \prime} \ominus k=k^{\prime \prime}-k$.

Theorem 2.11. For any $(p, q, t) \in \mathrm{H}_{1}$ and $(k, l, m) \in \mathrm{H}$, if $\left(k \Delta x, h l \Delta x, h m(\Delta x)^{2}\right) \approx(p, q, t)$, then

$$
\left\|\pi(W(k, l, m)) \mathbf{E}^{\star} f-{ }^{\star}\left(\rho_{h}(p, q, t) f\right)\right\|_{2} \approx 0
$$

for all $f \in L^{2}(\mathbf{R})$.

Proof. By the continuity of $\hat{\pi}(W(k, l, m))$ and $\rho_{h}(p, q, t)$ on $L^{2}(\mathrm{R})$, it suffices to prove Eq. (2.3) for all $f \in K(\mathrm{R})$. Let $f \in K(\mathrm{R})$. Suppose $\left(k \Delta x, h l \Delta x, h m(\Delta x)^{2}\right) \approx$ $(p, q, t)$. Since $f \in K(\mathrm{R})$, there is a real $M>0$ such that ${ }^{\star} f(x)=0$ if $|x|>M$, and hence

$$
\mathbf{S} f=\sum_{-M<k^{\prime} \Delta x<M} f\left(k^{\prime} \Delta x\right) \Delta_{k^{\prime}}
$$

and

$$
\|\mathbf{S} f\|_{2}^{2}=\sum_{-M<k^{\prime} \Delta x<M}\left|f\left(k^{\prime} \Delta x\right)\right|^{2} \Delta x .
$$

Suppose $l \neq 0$. By the assumption, $l \Delta x=O(1)$, and hence

$$
1-\frac{\sin \pi(l \Delta x) \Delta x}{\pi(l \Delta x) \Delta x}=O\left(\frac{1}{K}\right) .
$$

Thus from Lemma 2.10,

$$
\begin{aligned}
\| & \pi(W(k, l, m)) \mathrm{S}^{\star} f-{ }^{*} \rho_{h}\left(k \Delta x, h l \Delta x, h m(\Delta x)^{2}\right) \mathrm{S}^{\star} f \|_{2}^{2} \\
= & (\Delta x)^{-1} \sum_{k^{\prime \prime} \in \mathrm{K}}\left|\left\langle\Delta_{k^{\prime \prime}}\left|\pi(W(k, l, m))-{ }^{\star} \rho_{h}\left(k \Delta x, h l \Delta x, h m(\Delta x)^{2}\right)\right| \mathrm{S}^{\star} f\right\rangle\right|^{2} \\
= & \left.(\Delta x)^{-1} \sum_{k^{\prime \prime} \in \mathrm{K}-M<k^{\prime} \Delta x<M} \sum^{\star} f\left(k^{\prime} \Delta x\right)\right|^{2} \times \\
& \times\left|\left\langle\Delta_{k^{\prime \prime}}\left|\pi_{h}(W(k, l, m))-{ }^{*} \rho_{h}\left(k \Delta x, h l \Delta x, h m(\Delta x)^{2}\right)\right| \Delta_{k^{\prime}}\right\rangle\right|^{2}
\end{aligned}
$$




$$
\begin{aligned}
& =(\Delta x)^{-1} \sum_{-M<k^{\prime} \Delta x<M}\left|{ }^{*} f\left(k^{\prime} \Delta x\right)\right|^{2}(\Delta x)^{2} O\left(\frac{1}{K}\right)^{2} \\
& =O\left(\frac{1}{K}\right)^{2} \sum_{-M<k^{\prime} \Delta x<M}\left|{ }^{*} f\left(k^{\prime} \Delta x\right)\right|^{2} \Delta x \\
& =O\left(\frac{1}{K}\right)^{2}\|\mathbf{S} f\|_{2}^{2} .
\end{aligned}
$$

Thus we have

$$
\left\|\pi(W(k, l, m)) \mathbf{S}^{\star} f-{ }^{\star} \rho_{h}\left(k \Delta x, h l \Delta x, h m(\Delta x)^{2}\right) \mathbf{S}^{\star} f\right\|_{2} \approx 0
$$

It is easy to see that the last relation holds for the case $l=0$ with equality. By Lemma 2.5 and Theorem 2.6, $\left\|\mathrm{E}^{\star} f-{ }^{\star} f\right\|_{2} \approx 0$, and hence $\left\|\pi(W(k, l, m))\left(\mathrm{E}^{\star} f-\mathrm{S}^{\star} f\right)\right\|_{2} \approx$ 0. By Lemma $2.5,\left\|\mathbf{S}^{\star} f-{ }^{\star} f\right\|_{2} \approx 0$, so that $\|{ }^{\star} \rho_{h}\left(k \Delta x, h l \Delta x, h m(\Delta x)^{2}\right)\left(\mathbf{S}^{\star} f-\right.$ $\left.{ }^{\star} f\right) \|_{2} \approx 0$. By strong continuity of $\rho_{h}$, we have $\|{ }^{\star} \rho_{h}\left(k \Delta x, h l \Delta x, h m(\Delta x)^{2}\right)^{\star} f-$ ${ }^{\star}\left(\rho_{h}(p, q, t) f\right) \|_{2} \approx 0$. Therefore, we have

$$
\begin{aligned}
&\left\|\pi(W(k, l, m)) \mathbf{E}^{\star} f-{ }^{\star}\left(\rho_{h}(p, q, t) f\right)\right\|_{2} \\
& \leq\left\|\pi_{h}(W(k, l, m))\left(\mathbf{E}^{\star} f-\mathrm{S}^{\star} f\right)\right\|_{2} \\
&+\left\|\pi(W(k, l, m)) \mathrm{S}^{\star} f-{ }^{\star} \rho_{h}\left(k \Delta x, h l \Delta x, h m(\Delta x)^{2}\right) \mathbf{S}^{\star} f\right\|_{2} \\
&+\left\|^{\star} \rho_{h}\left(k \Delta x, h l \Delta x, h m(\Delta x)^{2}\right)\left(\mathbf{S}^{\star} f-{ }^{\star} f\right)\right\|_{2} \\
&+\left\|^{\star} \rho\left(k \Delta x, h l \Delta x, h m(\Delta x)^{2}\right)^{\star} f-{ }^{\star}\left(\rho_{h}(p, q, t) f\right)\right\|_{2} \\
& \approx 0 .
\end{aligned}
$$

This completes the proof.

For any $(p . q . t) \in \mathbf{H}_{1}$, define $\Delta(p, q, t) \in \mathbf{H}$ by

$$
{ }^{\Delta}(p, q, t)=\left(\left[\frac{p}{\Delta x}\right],\left[\frac{q}{h \Delta x}\right],\left[\frac{t}{h(\Delta x)^{2}}\right]\right)
$$

where $[r]$ is the greatest integer $n$ such that $n \leq r$. Recall that $L^{2}(\mathrm{R})$ is embedded in $\hat{L}^{2}(\mathcal{F})$ by the realation $f=\hat{\mathbf{E}} f$ for all $f \in L^{2}(\mathbf{R})$. Then we have the following. 
Theorem 2.12. For any $(p, q, t) \in \mathbf{H}_{1}$ and $f \in L^{2}(\mathbf{R})$,

$$
\hat{\pi}\left(W\left({ }^{\Delta}(p, q, t)\right)\right) f=\rho_{h}(p, q, t) f .
$$

Proof. Let $f \in L^{2}(\mathrm{R})$, and $(k, l, m)=\Delta(p, q, t)$. Since $\left(k \Delta x, h l \Delta x, h m(\Delta x)^{2}\right) \approx$ $(p, q, t)$, from Theorem 2.11,

$$
\begin{aligned}
\| \hat{\pi} & \left(W\left({ }^{\Delta}(p, q, t)\right)\right) f-\rho_{h}(p, q, t) f \|_{2} \\
& ={ }^{\circ} \| \pi_{h}\left(W\left({ }^{\Delta}(p, q, t)\right) \mathbf{E}^{\star} f-{ }^{\star}\left(\rho_{h}(p, q, t) f\right) \|_{2}\right. \\
& =0 .
\end{aligned}
$$

Let $\operatorname{fin}(\mathrm{H}, \Delta x)$ and $\mu(\mathrm{H}, \Delta x)$ be such that

$$
\begin{aligned}
\operatorname{fin}(\mathrm{H}, \Delta x) & =\left\{(k, l, m) \mid\left(k \Delta x, l \Delta x, m(\Delta x)^{2}\right) \in \mathrm{fin}\left(\mathrm{H}_{1}\right)\right\} \\
\mu(\mathrm{H}, \Delta x) & =\left\{(k, l, m) \mid\left(k \Delta x, l \Delta x, m(\Delta x)^{2}\right) \in \mu\left(\mathrm{H}_{1}\right)\right\}
\end{aligned}
$$

Then it follows from a similar argument to the proof of Lemma 2.9 that fin $(\mathrm{H}, \Delta x)$ is a subgroup of $\mathbf{H}$, and $\mu(\mathbf{H}, \Delta x)$ is a normal subgroup of $f(n)(H, \Delta x)$. By Lemma 2.7, the quotient group $\operatorname{fin}(\mathrm{H}, \Delta x) / \mu(\mathrm{H}, \Delta x)$ is isomorphic to $\mathrm{H}_{1}$. Thus we have the following theorem.

Theorem 2.13. Let $f \in L^{2}(\mathrm{R})$ with $f \neq 0$, and $\mathcal{H}$ be the closed subspace of $\hat{R}(\mathrm{~K}, \Delta x)$ such that

$$
\mathcal{H}=\left\{\hat{W}(k, l, m)\left({ }^{\circ} \mathcal{E}^{\star} f\right) \mid(k, l, m) \in \operatorname{fin}(\mathbf{H}, \Delta x)\right\}^{\perp \perp}
$$

For any $(k, l, m) \in \operatorname{fin}(\mathrm{H}, \Delta x)$, let $\tilde{W}(k, l, m)$ be the restriction of $\hat{W}(k, l, m)$ to $\mathcal{H}$. Then the map $(p, q, t) \mapsto \tilde{W}\left({ }^{\Delta}(p, q, t)\right)$ is a strongly continuous unitary representation of $\mathrm{H}_{1}$ unitarily equivalent to the Schrödinger representation $\rho_{h}$ on $L^{2}(\mathrm{R})$.

Proof. An immediate consequence from Theorem 2.12 and the irreducibility of $\rho_{h}$. 


\subsection{Noncommutative Parseval's identity}

In [25], one of the authors (I.O.) proposed an idea of a nonstandard-analytic proof of noncommutative Parseval's identity for the Schrödinger representation of the CCR by transferring the orthogonality relations for unitary representations of compact or finite groups. The rigorous proof along with this line can be carried out in the present framework. The proof runs as follows.

Noncommutative Parseval's identity for the Schrödinger representation of the CCR is known as the following relation

$$
\iint_{\mathbf{R}^{2}}\left\langle\psi_{1}\left|\rho_{h}(p, q, 0)\right| \varphi_{1}\right\rangle\left\langle\varphi_{2}\left|\rho_{h}(p, q, 0)^{-1}\right| \psi_{2}\right\rangle \frac{d p d q}{h}=\left\langle\varphi_{2} \mid \varphi_{1}\right\rangle\left\langle\psi_{1} \mid \psi_{2}\right\rangle
$$

for all $\psi_{i}, \varphi_{i} \in L^{2}(\mathrm{R})(i=1,2)$.

By the way, let $G$ be a compact group with two-sided Haar measure $\mu, \sigma$ an irreducible unitary representation of $G$ on a unitary space $V$. Then the following orthogonality relations hold

$$
\int_{G}\left\langle\psi_{1}|\sigma(g)| \varphi_{1}\right\rangle\left\langle\varphi_{2}\left|\sigma(g)^{-1}\right| \psi_{2}\right\rangle d \mu(g)=\frac{\mu(G)}{\operatorname{dim}(V)}\left\langle\varphi_{2} \mid \varphi_{1}\right\rangle\left\langle\psi_{1} \mid \psi_{2}\right\rangle
$$

for any $\varphi_{i}, \psi_{i} \in L^{2}(G)(i=1,2)$. By Transfer Principle, the internal irreducible representation $W(=\sigma)$ of the hyperfinite group $\mathrm{H}(=G)$ on the space $R(\mathbf{K}, \Delta x)$ $(=V)$ satisfies the above relation with $\mu(G)=K^{3}$ and $\operatorname{dim}(V)=K$. Thus we have

$$
\frac{1}{K^{2}} \sum_{k, l, m \in \mathbf{K}}\left\langle\psi_{1}|W(k, l, m)| \varphi_{1}\right\rangle\left\langle\varphi_{2}\left|W(k, l, m)^{-1}\right| \psi_{2}\right\rangle=\left\langle\varphi_{2} \mid \varphi_{1}\right\rangle\left\langle\psi_{1} \mid \psi_{2}\right\rangle
$$

for all $\varphi_{i}, \psi_{i} \in R(K, \Delta x)(i=1,2)$. From the relation $W(k, l, m)=e^{2 \pi i m / K} W(k, l, 0)$ and $W(k, l, m)^{-1}=e^{-2 \pi i m / K} W(k, l, 0)^{-1}$, we have

$$
\frac{1}{K} \sum_{k, l \in \mathrm{K}}\left\langle\psi_{1}|W(k, l, 0)| \varphi_{1}\right\rangle\left\langle\varphi_{2}\left|W(k, l, 0)^{-1}\right| \psi_{2}\right\rangle=\left\langle\varphi_{2} \mid \varphi_{1}\right\rangle\left\langle\psi_{1} \mid \psi_{2}\right\rangle
$$


Since $W(k, l, m)$ and $\pi(W(k, l, m))$ are internally unitarily equivalent, we have

$$
\sum_{k, l \in \mathbf{K}}\left\langle\psi_{1}|\pi(W(k, l, 0))| \varphi_{1}\right\rangle\left\langle\varphi_{2}\left|\pi\left(W(k, l, 0)^{-1}\right)\right| \psi_{2}\right\rangle(\Delta x)^{2}=\left\langle\varphi_{2} \mid \varphi_{1}\right\rangle\left\langle\psi_{1} \mid \psi_{2}\right\rangle
$$

for all $\varphi_{i}, \psi_{i} \in L^{2}(\mathcal{F})(i=1,2)$.

Let $\mathcal{D}(\mathbf{R})$ be the space of infinitely differentiable complex-valued functions on $\mathbf{R}$ with compact support. By the density of $\mathcal{D}(\mathrm{R})$ in $L^{2}(\mathbf{R})$, we can assume $\varphi_{i}, \psi_{i} \in$ $\mathcal{D}(\mathrm{R})(i=1,2)$ without any loss of generality. Then there is a standard $M>0$ such that ${ }^{\star} \varphi_{i}(x)={ }^{\star} \psi_{i}(x)=0$ for all $x \in{ }^{\star} \mathrm{R}$ with $|x|>M$. Thus we have for any $k, k^{\prime} \in \mathrm{K},{ }^{\star} \varphi_{i}\left(\left(k^{\prime} \oplus k\right) \Delta x\right)=\varphi_{i}\left(k^{\prime} \Delta x+k \Delta x\right)$, etc.

Then, from Eq. (2.6) and Lemma 2.5, we have

$$
\begin{aligned}
& \left\langle\varphi_{2} \mid \varphi_{1}\right\rangle\left\langle\psi_{1} \mid \psi_{2}\right\rangle \\
& \approx\left\langle\mathrm{S}^{\star} \varphi_{2} \mid \mathrm{S}^{\star} \varphi_{1}\right\rangle\left\langle\mathrm{S}^{\star} \psi_{1} \mid \mathrm{S}^{\star} \psi_{2}\right\rangle \quad \text { (by Lemma } 2.5 \text { ) } \\
& =\sum_{k, l \in \mathbf{K}}\left\langle\mathrm{S}^{\star} \psi_{1}|\pi(W(k, l, 0))| \mathrm{S}^{\star} \varphi_{1}\right\rangle\left\langle\mathrm{S}^{\star} \varphi_{2}\left|\pi\left(W(k, l, 0)^{-1}\right)\right| \mathrm{S}^{\star} \psi_{2}\right\rangle(\Delta x)^{2} \quad \text { (by Eq. (2.6) } \\
& =\sum_{k, l, k^{\prime}, k^{\prime \prime} \in \mathrm{K}} \overline{{ }^{*} \psi_{1}\left(k^{\prime} \Delta x\right)} e^{2 \pi i(l \Delta x)\left(k^{\prime} \Delta x\right) \star} \varphi_{1}\left(k^{\prime} \Delta x+k \Delta x\right) e^{-2 \pi i(l \Delta x)\left(k^{\prime \prime} \Delta x\right)} \times \\
& \times^{\star} \varphi_{2}\left(k^{\prime \prime} \Delta x+k \Delta x\right)^{\star} \psi_{2}\left(k^{\prime \prime} \Delta x\right)(\Delta x)^{4} \quad \text { (by definition) } \\
& \approx \int_{\star_{\mathrm{R}^{4}}} \overline{{ }^{*} \psi_{1}\left(x^{\prime}\right)} e^{2 \pi i q x^{\prime} / h \star} \varphi_{1}\left(x^{\prime}+p\right) e^{-2 \pi i q x^{\prime \prime} / h} \times \\
& \times^{\star} \varphi_{2}\left(x^{\prime \prime}+p\right){ }^{\star} \psi_{2}\left(x^{\prime \prime}\right) d p(d q / h) d x^{\prime} d x^{\prime \prime} \quad \text { (by Lemma 2.5) } \\
& =\int_{\mathbf{R}^{2}}\left\langle\psi_{1}\left|\rho_{h}(p, q, 0)\right| \varphi_{1}\right\rangle\left\langle\varphi_{2}\left|\rho_{h}(p, q, 0)^{-1}\right| \psi_{2}\right\rangle \frac{d p d q}{h} \text {. (by definition) }
\end{aligned}
$$

Since the first and the last terms are standard, they must be equal. Therefore, we have proved Eq. (2.4) from Eq. (2.5).

\section{Logical extension methods in physics}

The purpose of this section is to give a brief account on the present status and the achievement of the program in the logical extension methods in physics, which is 
summarized in Figure 1.

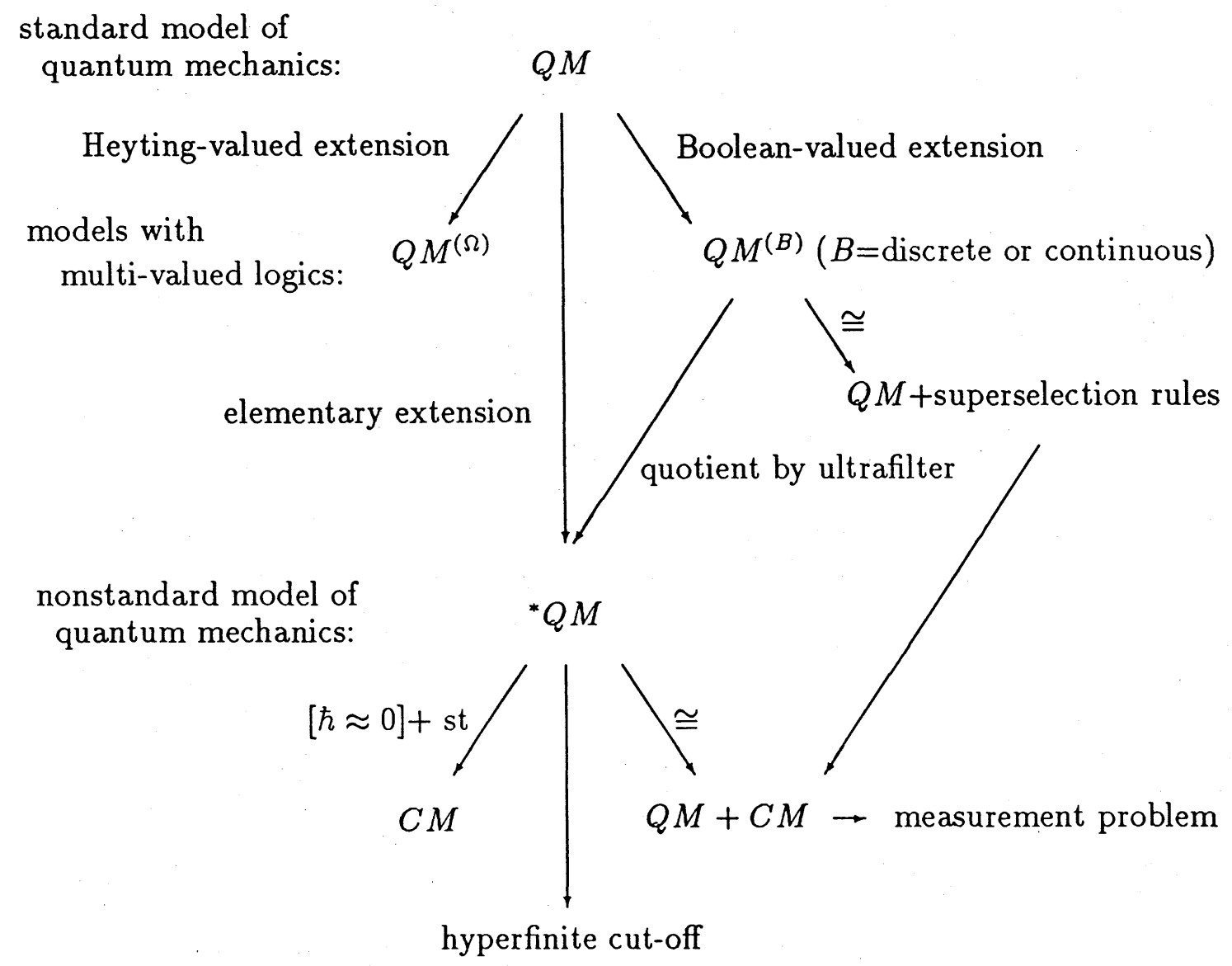

Figure 1: Diagram of logical extension methods. Every site shows a physical theory, and every arrow shows a method for obtaining a new theory or a relation between two theories.

\subsection{Boolean-valued extension of quantum mechanics}

Throughout this section a "Boolean algebra" means a "complete Boolean algebra" in the ordinary sense, and $B$ denotes a Boolean algebra. In the diagram, $Q M$ stands for the standard quantum mechanics and $C M$ the standard classical mechanics. 
The Boolean-valued universe $V^{(B)}$ of set theory based on $B$ is a model of ZFC, the Zermelo-Fraenkel set theory with the Axiom of Choice, in which the truth value of a statement is determine as an element of $B$ [13]. In the right side, $Q M^{(B)}$ stands for the "quantum mechanics" in the Boolean-valued universe, or Boolean-valued quantum mechanics in short, which is the interpretation of syntactical rules of quantum mechanics in a Boolean-valued universe instead of the standard universe. The arrow from $Q M$ to $Q M^{(B)}$ symbolizes the process of Boolean-valued extension, one of the logical extensions, of quantum mechanics, that is the change of the theory from standard quantum mechanics to the Boolean-valued quantum mechanics.

A common feature of such logical extensions is to leave the syntax of the original theory invariant, but to change the semantics according to certain logical procedures described rigorously. Since the validity of mathematical inferences or proofs is dominated only by syntactical rules as formulated in terms of symbolic manipulations, and is independent of the meanings of sentences, the change of semantics without changing the syntax does not affect the syntactical validity such as provability or deducibility, and leads to transfer principles which transfer a valid sentence in one theory to the corresponding valid sentence in another. Nevertheless, the extended theory can have the drastically different physical meaning yielded by the semantical change.

The peculiar feature of the Boolean-valued extension is the change of logic from the 2 -valued logic to the $B$-valued logic, a certain many-valued logic with even continuously many truth values. A serious analysis of the Boolean-valued quantum mechanics arose as the first attempt of the logical extension methods in the following circumstances.

In 1980 Machida and Namiki [20] proposed a theory of measurement, called the many Hilbert space theory, incorporating with extending the formalism of quantum 
mechanics by allowing a macroscopic parameter, and Araki [21] laid the mathematical foundation of their extension on the continuous superselection rule (CSR). The arrow from " $Q M+$ superselection rules" to "QM+CM" symbolizes Araki's proposal of using the CSR as the framework of description of micro-macro combined systems. In 1984 Yanase [30] pointed out a parallelism between an idea of the indefinite description of a quantum system in Machida-Namiki theory and fuzzy set theory, and proposed a program of making foundations of Machida-Namiki theory from the logical point of view. By the way, in 1983 Takeuti [17] and Ozawa [18] established the transfer principle between "the algebras of all bounded operators on Hilbert spaces" in a Boolean-valued universe and "the type I von Neumann algebras of all decomposable operators on direct integral Hilbert spaces" in the standard universe. From this result, Ozawa [22] established the transfer principle between the Boolean-valued quantum mechanics and the standard quantum mechanics with a CSR. According to this transfer principle, we have the following equivalence between the two theories.

$$
Q M+C S R_{\mu}=Q M^{(B)}+\text { "averaging by } \mu \text { ", }
$$

where $C S R_{\mu}$ is the continuous superselection rule induced by an absolutely continuous measure $\mu$, and $B$ is chosen as the measure algebra of $\mu$. The arrow from $Q M^{(B)}$ to " $Q M+$ superselection rule" symbolizes this equivalence.

Thus the consecutive three arrows from $Q M$ through $Q M^{(B)}$ to $Q M+C M$ give naturally a certain logical analysis of the Machida-Namiki theory. The real numbers in the Boolean-valued universe are represented by random variables, and hence the Boolean-valued quantum mechanics predicts the expectation of the outcome of a measurement as a random variable. Nevertheless, the prediction in $Q M+C S R_{\mu}$ is a standard real number, which in turn coincides with the average of the prediction in $Q M^{(B)}$ by measure $\mu$. Thus we can conclude that the semantical change from the 
$Q M$ to $Q M+C S R_{\mu}$ is the change of logic from 2-valued logic to the Boolean-valued logic, and that the syntactical change is the additional prescription of averaging the random prediction by measure $\mu$. This kind of understanding of the CSR leads to the following answer to Yanase's problem: the Machida-Namiki extension of quantum mechanics by adding a macroscopic parameter is mathematically equivalent to the extension of the real number system augmented by a generic real by the method of forcing. For the full account of the parallelism between the Machida-Namiki theory and Cohen's forcing, we will refer to [22].

\subsection{Heyting-valued extension of quantum mechanics}

Heyting algebras naturally arise as the algebraic structure of the truth values in the intuitionistic logic instituted by Brower. As the inference rules of the intuitionistic logic generalizes the classical logic, the Heyting algebras generalize the Boolean algebras. The most typical example of a Heyting algebra is the complete lattice of open sets in a topological space. The construction of the Heyting-valued universe is a direct adaptation of the construction of the Boolean-valued universe, and the Heytingvalued universe is a model of intuitionistic set theory, topos theory, and higher-order sheaf theory as well as fuzzy set theory in a special case [31]. However, "the quantum mechanics" in the Heyting-valued universe, indicated by $Q M^{(\Omega)}$ in the left side of the diagram, where $\Omega$ stands for a Heyting algebra, has not been studied seriously.

\subsection{Nonstandard extension of quantum mechanics}

The nonstandard universe is described in Subsection 2.1. The nonstandard quantum mechanics, denoted by ${ }^{\star} Q M$ in the center of the diagram, is the interpretation of quantum mechanics in the nonstandard universe. Since the nonstandard universe is a bounded elementary extension of the standard superstructure, the nonstandard 
quantum mechanics is naturally an elementary extension of the standard quantum mechanics, as indicated by the arrow from $Q M$ to ${ }^{\star} Q M$. Usually, the nonstandard universe is constructed by the bounded ultrapower of a standard superstructure. However, Ozawa [32] extended the construction to any Boolean algebra $B$ so that the quotient of a "superstructure" in $V^{(B)}$ by an $\omega$-incomplete ultrafilter yields a nonstandard universe, and that the ordinary construction corresponds a special case where $B$ is atomic. The arrow from $Q M^{(B)}$ to ${ }^{\star} Q M$ indicates the change from $Q M^{(B)}$ to ${ }^{\star} Q M$ caused by this construction of the nonstandard universe. Thus, $Q M^{(B)}$ has another way to yield a theory in the 2 -valued logic with numerical predictions. The arrows from $Q M^{(B)}$ to $Q M+C M$ via ${ }^{\star} Q M$ corresponds in turn to selecting an ideal superselection sector contrasting to the way of averaging over all superselection sectors mention in Subsection 3.1. However, these two theories obey entirely different physical interpretations on these numericalization processes. The arrow from ${ }^{\star} Q M$ to $C M$ indicate the process of choosing the unit with $\hbar \approx 0$ and taking the standard part, which is equivalent to taking limit as $\hbar \rightarrow 0$ in the traditional correspondence principle. Thus the process indicated by this arrow yields the classical mechanics. The arrow from ${ }^{\star} Q M$ to $Q M+C M$ indicates that the nonstandard quantum mechanics gives rise to a synthesis of quantum mechanics and classical mechanics in a natural way. In the nonstandard quantum mechanics, the observables are represented by the internal self-adjoint operators on an internal Hilbert space $\mathcal{H}$, and the states the internal density operators. Obviously, the expectation values are predicted as hyperreal numbers, and hence we will call the standard part of a prediction of ${ }^{\star} \mathrm{QM}$ as the standardized prediction.

The case where the quantum system is an one-dimensional quantum harmonic oscillator is examined in detail by Ozawa [6]. In this case, by direct transfer we have $\mathcal{H}=L^{2}\left({ }^{\star} \mathrm{R}\right)$, and the Hamiltonian is $H=\hbar \omega\left({ }^{\star} N+\frac{1}{2}\right)$, where $N$ is the internal 
number operator and $\omega$ is the angular frequency. Now, we have options in choosing a unit system in describing the same physical system; the following two unit systems are considered: 1) a micro-unit system in which $\hbar=1$, and 2) a macro-unit system in which $\hbar=\epsilon$ where $\epsilon \approx 0$, and the units of mass and time is assumed to be the same in the both scales. Then under this assumptions, it is shown that the standardized prediction of ${ }^{\star} Q M$ under the micro-unit system is the same as the standard quantum mechanics, whereas the standardized prediction under the macrounit system coincides with the classical harmonic oscillator with the same mass and angular frequency. Thus we have

$$
\begin{aligned}
& { }^{\star} Q M+" \hbar \sim 1 "+\text { "standard part" }=\mathrm{QM}, \\
& { }^{\star} Q M+" \hbar \approx 0 "+" \text { standard part" }=\mathrm{CM} .
\end{aligned}
$$

In this way, we have in ${ }^{\star} \mathrm{QM}$ a consistent synthesis of quantum mechanics and classical mechanics described by the same mathematical structure with different unit systems. For the detail. we refer to [6].

\subsection{Hyperfinite cut-off}

In the ordinary physics, the cut-off is used to avoid the divergence in the early stage of calculations. However, it is usual that a theory with the cut-off and the theory after the limit process of clearing the cut-off are much different in mathematical or intuitive point of view. In the nonstandard quantum mechanics, the hyperfinite cut-off is possible, and taking the standard part clears the cut-off. Thus the theories with cutoff and without cut-off can describe the same physical state; this is usually impossible in the ordinary approach since the taking of the limit changes the physical state described by the theory. Our approach of the hyperfinite Heisenberg group developed in Section 2 is considered as a unified approach to the hyperfinite cut-off theories of the 
nonstandard quantum mechanics. In fact, the Schrödinger representation discussed in Subsection 2.4 is equivalent to the hyperfinite cut-off of both position and momentum, and the number-phase quantization proposed in [23] corresponds to the hyperfinite cut-off of the number of quanta. The arrow from * $Q M$ to the "hyperfinite cut-off" is slightly more open than others, and prompting a future development of this direction.

\section{Acknowledgments}

One of the authors (M.O.) benefited from the visiting membership system at RIMS, Kyoto University, which facillitated our collaboration. This work was partially supported by JSPS grants (Nos.*** and ${ }^{* * *}$ ).

\section{References}

[1] Proc. 1st, 2nd, and 3rd Int. Symp. Foundations of Quantum Mechanics. Japan Society of Physics, Tokyo, $(1983,1986,1989)$. P. Tombesi and E. R. Pike, editors. Squeezed and Nonclassical Light. Plenum, New York, 1989. O. Hirota C. Bendjaballah and S. Reynaud, editors. Proc. Int. Workshop on Quantum Aspects of Optical Communications. Lecture Notes in Physics No.3r8, Springer-Verlag, Berlin, (1991). L. Smarr, editor. Sources of Gravitational Radiation. Cambridge UP, Cambridge, 1979. P. Meystre and M. O. Scully, editors. Quantum Optics, Experimental Gravitation and Measurement Theory. Plenum, New York, 1983.

[2] E. B. Davies. Quantum Theory of Open Systems. Academic Press, London, 1976. C. W. Helstrom. Quantum Detection and Estimation Theory. Academic Press, New York, 1976. A. S. Holevo. Probabilistic and Statistical Aspects of Quantum Theory. North-Holland, Amsterdam, 1982. M. Ozawa. J. Math. Phys., 25, 7987, (1984); 26, 1948-1955, (1985); 27, 759-763, (1986). Publ. RIMS, Kyoto Univ., 21, 279-295, (1985); P. J. Lahti P. Busch and P. Mittelstaedt. The 
Quantum Theory of Measurement. Lecture Notes in Physics, Springer-Verlag, Berlin, 1991.

[3] H. P. Yuen and J. H. Shapiro. IEEE Trans. Inf. Theory, IT-26, 78, (1984). C. M. Caves, K. S. Throne, R. W. P. Drever, V. D. Sandberg, and M. Zimmermann. Rev. Mod. Phys, 52, 341, (1980). H. P. Yuen. Phys. Rev. Lett, 51, 719-722, (1983). M. Ozawa. Phys. Rev. Lett., 60, 385-388, (1988); 67, 1956-1959, (1991).

[4] O. Bratteli and D. W. Robinson. Operator Algebras and Quantum Statistical Mechanics I. Springer-Verlag, New York, 1979; II. 1979.

[5] M. Ozawa. Cat Paradox for $C^{*}$-dynamical Systems. Preprint Series, Dept. Math., Coll. Gen. Ed., Nagoya University, 1992.

[6] M. Ozawa. Infinitesimal analysis and the measurement problem (in Japanese). In Soryuushi-ron Kenkyuu; Prog. Theor. Phys. (in preparation).

[7] R.C. Tolman. The Principles of Statistical Mechanics. Oxford University Press, Inc., 1938; (Dover edition) Dover Publications, Inc., 1979.

[8] I.M. Lifshitz and L.P. Pitaevskii. Landaru and Lifshitz: Course of Theoretical Physics Vol.5 Statistical Physics Part 1 (3rd edition) (English edition) Pergamon Press, 1976

[9] A. Robinson. Non-Standard Analysis. North-Holland, Amsterdam, 1966.

[10] K. D. Stroyan and W. A. J. Luxemburg. Introduction to the Theory of Infinitesimals. Academic Press, New York, 1976. M. Davis. Applied Nonstandard Analysis. Wiley, New York, 1977. A. E. Hurd and P. A. Loeb. An Introduction to Nonstandard Real Analysis. Academic Press, Orland, 1985.

[11] H. J. Keisler. Mem. Amer. Math. Soc., 48, No. 297, 1984. K. D. Stroyan and J. M. Bayod. Foundations of Infinitesimal Stochastic Analysis. North-Holland, 
Amsterdam, 1986. S. Albeverio et. al. Nonstandard methods in stochastic analysis and mathematical physics. Academic, Orland, 1986.

[12] P. J. Cohen. Set Theory and the Continuum Hypothesis. Benjamin, New York, 1966.

[13] J. L. Bell. Boolean-Valued Models and Independence Proofs in Set Theory. 2nd ed. Oxford University Press, Oxford, 1985.

[14] D. Scott. In Applications of Model Theory to Algebra, Analysis, and Probability, Holt, Reinhart and Winston, 1969.

[15] G. Takeuti. Two Applications of Logic to Mathematics. Princeton University Press, Princeton, 1978.

[16] G. Takeuti. J. Symbolic Logic, 44, 417-440, (1979); Japan. J. Math., 9, 207-245, (1983). M. Ozawa. J. Math. Soc. Japan, 36, 589-608, (1984); J. London Math. Soc. (2), 32, 141-148, (1985); 33, 347-354, (1986). K. Smith. J. Symbolic Logic, 49, 281-297, (1984). H. Nishimura. J. Symbolic Logic, 56, 731-741, (1991).

[17] G. Takeuti. J. Math. Soc. Japan, 35, 1-21, (1983)

[18] M. Ozawa. J. Math. Soc. Japan, 35, 609-627, (1983).

[19] T. Hinokuma and M. Ozawa. Preprint Series 6, Dept. Math., Coll. Gen. Ed., Nagoya University, 1986; Ill. J. Math., (to appear).

[20] S. Machida and M. Namiki. Prog. Theor. Phys., 63, 1457-1473, 1833-1847, (1980).

[21] H. Araki. Prog. Theor. Phys., 64, 719-730, (1980).

[22] M. Ozawa. Journal of the Japan Association for Philosophy of Science, 18, $35-43,(1986)$. 
[23] M. Ozawa. In H. Araki, et. al. ed., Current Topics in Operator Algebras, pages 52-58, World Scientific, Singapore, 1991.

[24] D. T. Pegg and S. M. Barnett. Europhys. Lett., 6, 483-487,(1988); Phys. Rev. $A, 39,1665-1675$, (1989).

[25] I. Ojima. unpublished, 1991.

[26] P. A. Loeb. Trans. Amer. Math. Soc., 211, 113-122, (1975).

[27] G. Takeuti. Proc. Japan Acad., 38, 414-418, (1962). P. J. Kelemen and A. Robinson. J. Math. Phys., 13, 1870-1874, 1875-1878, (1972). R. Kambe. Prog. of Theor. Phys., 52, 688-706, (1974). M. O. Farrukh. J. Math. Phys., 16, 177200, (1975). Ph. Blanchard and J. Tarski. Acta Physica Austriaca, 49, (1978). R. Fittler. Helv. Phys. Acta, 57, 579-609, (1984); 60, 881-902, (1987).

[28] C. C. Chang and H. J. Keisler. Model Theory. Third edition. North-Holland, Amsterdam, 1990.

[29] G. B. Folland. Harmonic Analysis in Phase Space. Princeton UP, Princeton, - NJ, 1989.

[30] M. M. Yanase. Gendai Buturigaku to Atarashii Sekaizou [Modern Physics and New Outlook of the World] (in Japanese). Iwanami, Tokyo, 1984.

[31] G. Takeuti. Chokkan-Shugi-teki Sugoron [Intuitionistic Set Theory] (in Japanese). Kinokuni-ya, Tokyo, 1980.

[32] M. Ozawa. In Abstracts of the Special Session in Set Theory, American Mathematical Society, Honolulu, 1987. 